\title{
Advancing the care of patients with cerebrovascular disease: editorial
}

\author{
Tyler Scullen, Aaron S. Dumont \\ Department of Neurological Surgery, Tulane University, New Orleans, LA 70112, USA.
}

Correspondence to: Prof. Aaron S. Dumont, Department of Neurological Surgery, Tulane University, 131 South Robertson Street, Suite 1300, New Orleans, LA 70112, USA. E-mail: adumont2@tulane.edu

How to cite this article: Scullen T, Dumont AS. Advancing the care of patients with cerebrovascular disease: editorial. Vessel P/us 2020;4:9. http://dx.doi.org/10.20517/2574-1209.2020.07

Received: 4 Feb 2020 Accepted: 24 Feb 2020 Published: 10 Apr 2020

Science Editor: Alexander D. Verin Copy Editor: Jing-Wen Zhang Production Editor: Jing Yu

Contemporary understanding of cerebrovascular disease has seen rapid development coupled with translational clinical advancements. Increased knowledge of the basic processes of the disease has provided the basis for significant developments in the detection, prevention, and treatment of focal and systemic conditions whose combined effects present a disproportionately high burden on the health care system, associate with multiple significant co-morbidities, and often carry high and protracted morbidity and mortality ${ }^{[1]}$. Such trends have been the result of an increased progressive and coordinated effort in various related basic science and clinical fields. In this editorial, we review some important examples of the work providing multidisciplinary contributions to the understanding and treatment of Cerebrovascular Disease, as featured in this special issue.

Critical to the development of effective prevention and treatment strategies is an advanced understanding of underlying disease pathophysiology and molecular biology. Accordingly, Padarti et al ${ }^{[2]}$ provided a comprehensive and well written review on the epidemiology, cellular biology, and phenotypic expression of cerebral cavernous malformations (CCM) in the context of known CCM proteins. The article effectively describes disease biology in a stepwise fashion following a brief summary on present epidemiology and clinical presentation ${ }^{[2]}$. Current understanding of the structural biology and genotype of each individual implicated protein complex is provided prior to a discussion on consequent epigenetic processes and inter- and intracellular interactions ${ }^{[2]}$. A thoughtful review of possible mechanisms of disease is then presented, supported by current in vitro and in vivo data and anecdotal evidence ${ }^{[2]}$. Together, the authors provided a succinct and comprehensive report on our modern understanding of CCM pathobiology and suggested potential routes of future investigation. Such reviews are a vital substrate in the development of

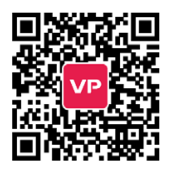


translational investigations and clinical advancement.

Along with an understanding of disease basic science, modern clinical practice mandates introspective evaluation of current practice. Such methods allow for the identification of areas of improvement in treatment and prevention strategies. Im et al. ${ }^{[3]}$ investigated the compliance and appropriate prescription of aspirin in the primary prevention of cerebrovascular and cardiovascular disease. The authors prospectively analyzed the baseline demographics of 1125 patients without prior vascular disease undergoing physicals with primary care physicians at their center ${ }^{[3]}$. Using patient demographics, the authors evaluated discrepancies in aspirin use at the time of their routine medical checkup ${ }^{[3]}$. The authors found that, while aspirin administration was indicated ${ }^{[4]}$ in $23.6 \%$ of patients, only $3.9 \%$ of patients were actively taking the $\operatorname{drug}^{[3]}$. Investigation of this discrepancy illustrated that only $10 \%$ of patients were appropriately prescribed aspirin based on current international guidelines, and thus through secondary calculation reasoned that $6 \%$ in total were taking the medicine appropriately ${ }^{[3]}$. The authors suggested that improvement in primary prevention may be made through increased education on aspirin use, at the level of both the prescriber and the patient ${ }^{[3]}$. Such studies provide opportunities in the prevention of complex cerebrovascular disease by highlighting areas where active guidelines are not being employed efficiently.

Similarly, effective selection of patients to undergo primary prevention of treatment measures allows for efficient resource management and decreases risks of adverse events. As such, growing interest in radiographic and molecular biomarkers has sparked interest in multiple aspects of cerebrovascular disease. In their prospective analysis, Mueller et al. ${ }^{[5]}$ sought to characterize radiographic features and various potential serum biomarkers and their association with clinical presentation of atherosclerotic carotid disease. Transcranial ultrasound bubble studies and radiotracer computer tomography angiography were utilized to evaluate the stability of plaque formation in symptomatic and asymptomatic patients with clinically significant carotid stenosis ${ }^{[5]}$. Given the uncertain nature regarding the stability of atherosclerotic plaques and their tendency to rupture and produce atheroembolic events, the subject has seen significant interest in active studies involving the evaluation of prognostic factors and underlying biology in plaque formation ${ }^{[6]}$. The authors chose to compare important clinical indicators, such as propensity of microemboli formation, with biomarkers under investigation, concluding that ICAM-1 was associated with thrombotic plaque formation ${ }^{[5]}$.

As an adjunct to active research, case reports provide valuable anecdotal evidence of the variable presentation and management techniques of disease. Candelaresi $e t$ al ${ }^{[7]}$ presented a well written report of a patient who developed posterior reversible encephalopathy syndrome attributable to the administration of the myeloma protease inhibitor bortezomib. The case details the clinical course and association with drug and discusses potential mechanisms ${ }^{[5]}$. The patient in the case was ultimately successfully treated with systemic glucocorticoids ${ }^{[5]}$, a convenient option as such drugs are often a component of anti-myeloma drug regimens.

Cerebrovascular disease has undergone a recent and synergistic increase in disease understanding and treatment options. Such phenomena are only possible with continued and persistent multidisciplinary academic interest. Critical literature takes a variety of forms. Literature reviews provide a concise and relevant presentation of basic science knowledge of disease processes and may provide functional benefit to other basic scientists, clinicians, and translational scientists. Introspective evaluations of current disease practices allow quality improvement and an effective utilization of active management strategies. Studies identifying and validating disease biomarkers are increasingly necessary given the heterogeneous nature of multiple disease processes to assure effective patient selection and resource allocation. Finally, case reports allow investigators to be aware of variations of disease presentations and effects of seemingly unrelated events to provide anecdotal evidence that may affect clinical decision making or inspire connections in 
scientific hypotheses. This special issue of Vessel Plus provides valuable contributions for those investigating cerebrovascular disease and treating patients harboring disease of the cerebral circulation.

\section{DECLARATIONS}

Authors' contributions

Drafting the work: Scullen T

Substantial contributions to the conception or design of the work, revised the work critically for important intellectual content, and made final approval of the version to be published: Dumont A

Agreement to be accountable for all aspects of the work in ensuring that questions related to the accuracy or integrity of any part of the work are appropriately investigated and resolved: Scullen T, Dumont A

\section{Availability of data and materials}

Not applicable.

\section{Financial support and sponsorship}

None.

\section{Conflicts of interest}

Both authors declared that there are no conflicts of interest.

\section{Ethical approval and consent to participate}

Not applicable.

\section{Consent for publication}

Not applicable.

\section{Copyright}

(c) The Author(s) 2020.

\section{REFERENCES}

1. Ng SH, Rahman N, Ang IYH, Sridharan S, Ramachandran S, et al. Characterization of high healthcare utilizer groups using administrative data from an electronic medical record database. BMC Health Serv Res 2019;19:452.

2. Padarti A, Zhang J. Recent advances in cerebral cavernous malformation research. Vessel Plus 2018;2:21.

3. Im JH, Han SW, Lee SY, Baik JS. Adherence to guidelines: primary prevention with aspirin in 1125 medical check-up participants. Vessel Plus 2018;2:5.

4. Bibbins-Domingo K; US Preventive Services Task Force. Aspirin use for the primary prevention of cardiovascular disease and colorectal cancer: US preventive services task force recommendation statement. Ann Intern Med 2016;164:836-45.

5. Mueller H, Fisch L, Bonvin C, Lovblad K, Ratib O, et al. Plasmatic biomarkers of inflammation correlate with 18FDG-PET-CT and microembolic signals in patients with carotid stenosis. Vessel Plus 2018;2:13.

6. Kan Y, He W, Ning B, Li H, Wei S, et al. The correlation between calcification in carotid plaque and stroke: calcification may be a risk factor for stroke. Int J Clin Exp Pathol 2019;12:750-8.

7. Candelaresi P, Casorio MC. Bortezomib-induced posterior reversible encephalopathy syndrome: a case report. Vessel Plus 2018;2:11. 